\title{
Sleep Monitoring System
}

\author{
Achuth Rahul \\ School of Computing Science \\ and Engineering \\ VIT University \\ Chennai INDIA
}

\author{
Kathir Deivanai \\ School of Computing Science and \\ Engineering \\ VIT University \\ Chennai INDIA
}

\begin{abstract}
Today world is seeking for immediate testing kit with support of close notification to user via their mobile phones or through the remote wireless node instead of always consulting with physician. Necessity of this kind of systems for emergency treatment basis, supports the life saving tasks for anyone whoever facing the problem like sleep disorders. Distribution of framework within the embedded system for monitoring of patient's vital functions, such as pulse rate. By the usage of ARM microcontroller, the system will determine that the sleeping nature of the persons. For Emergency needs the monitoring device will send alarm. Main focus of the project, to accomplish the Emergency health care scheme even when acute disease breaks suddenly at nights or day and provide safety, healthy and quality of life of people. Drowsiness is also a big public safety hazard every day on the road .This project helps to provide alerts to driver, give caution to workers who works late night and also passes over the information to preset emergency call centre like 100. So that preliminary medical treatment can be provided without risking the life of the person. Advantage of the scheme is to satisfy the patient's requirement through the remote mechanism as well as ensure of low cost.
\end{abstract}

\section{INTRODUCTION}

To face the challenges in present day situation, the humans wish to lead their lives with automated systems instead of manual operations. The rapid growth of embedded technology helps in various domains such as smart environment, pervasive computing, energy management, cyber security, industry automation, automobile, online electronic payment, aeronautics and telemedicine. In order to monitor sleep of the patient's there is need of awareness about new bio-sensor and wireless technology. Here the work is divided into two sections, in which we are monitoring the patient vital values and sending the information to the diagnostics via zigbee protocol and pass over the particular information's(medical records) to their mobile phones via gsm modem. Development of real bio-telemetric system, the project is created with the shelf embedded hardware technology, namely ARM microcontrollers, Flash RAM memory and dedicated ZigBee chips. Measurement of activities of a patient also plays a major role. The information pass over via our system must be reliable and continuous.

\section{RELEATED WORK}

Sleep is an important physiological phenomenon [1], if a person has a good sleep he can be more active and he will be gaining more capability to do works faster and grasp the things very easily. If the sleep disturbs it will directly affect our work, life and even cause the disease. So there is requirement of automated system through which we can process the data with in short time and alert them. So Bluetooth based automated system [2] has been developed which provide a limited coverage area and more cost. Sleep monitoring system[1] is sensing the value and blow alarm when the sensed value cross the normal range. But it is only sensing whether the person suffers acute disease or not. . we developed a automated system which provide more coverage and cost is within range. It has capability to monitor and provide alerts to drivers and caution to workers who work in late nights.

\section{CONCEPTS OF BIOTELEMETRIC SYSTEM}

Model bio-telemetric system can be partitioned in to two parts. Inner part located in patient's home and outer part present in monitoring center. Both parts are again sub partitioned into participating elements

\subsection{Inner part of model Bio-telemetric System}

There are two main hardware elements in inner part of model bio-telemetric system; each one is marked with a symbolic name

- $\quad$ stationary data acquiring unit

- $\quad$ stationary data forwarding unit

Sensors are stationary dedicated units. Each sensor has dedicated task to do. Measurement of these values is not always fully automatic and requires certain cooperation from patient. The measurement of pulse rate is done depending on specialized sensor hardware. ORTHRUS is a stationary data forwarding unit. It gathers various measured data from elements of inner part of model bio-telemetric system and forwards them to outer part of this system. ORTHRUS will acts as midpoint between inner part of model bio-telemetric system and outer part of system. For Valid pulse measurement therefore requires extensive pre-processing of the raw signal.

The new signal processing approach presented here combines analog and digital signal processing in a way that, both parts can be kept simple but in combination, they are very effective in suppressing disturbance signals.

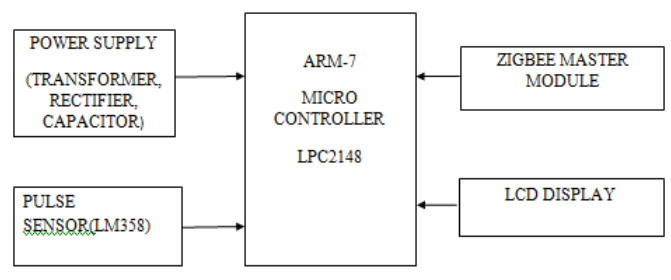

Fig 1: Block diagram of patient section 


\subsection{Outer Part of Model Bio-telemetric system}

By using zigbee protocol the information is received to the outer part. Information is then processed and analyzed. If the analyzed data crosses the pre set threshold value the buzzer gives the alarm and Passover the information to respected person

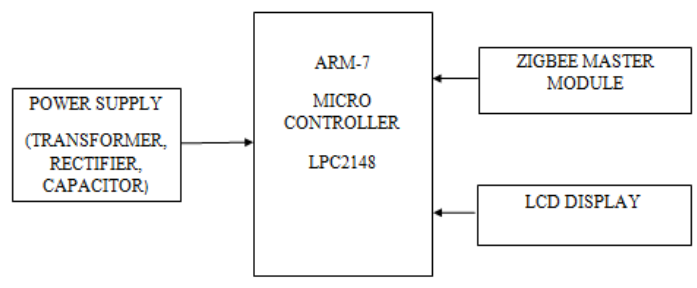

Fig 2: Block diagram of doctor section

\section{KEY DATA PROCESSING HARDWARE COMPONENT}

\subsection{Arm controller}

In this project the micro-controller plays major role. The micro controller used is ARM7(lpc2148). It is a 32 bit processor core, used for high end application. ARM stands for Advanced RISC machines. It has $8 \mathrm{~kb}$ to $40 \mathrm{~kb}$ of on-chip static RAM and $32 \mathrm{~kb}$ to $512 \mathrm{~kb}$ of on chip flash memory. Primary purpose of this cache is to provide temporary data storage in case of short time ZigBee or Ethernet network failure. It has one 10bit ADC with conversion time less than 2.44 microseconds per channel and one 10bit DAC also. Generally Micro-controllers were originally used as components in complicated process-control systems. Because of their small size and low cost, Micro-controllers are now also being used in regulators for individual control loops. In numerous areas Micro-controllers are now outperforming their analog counterparts and are cheaper as well.

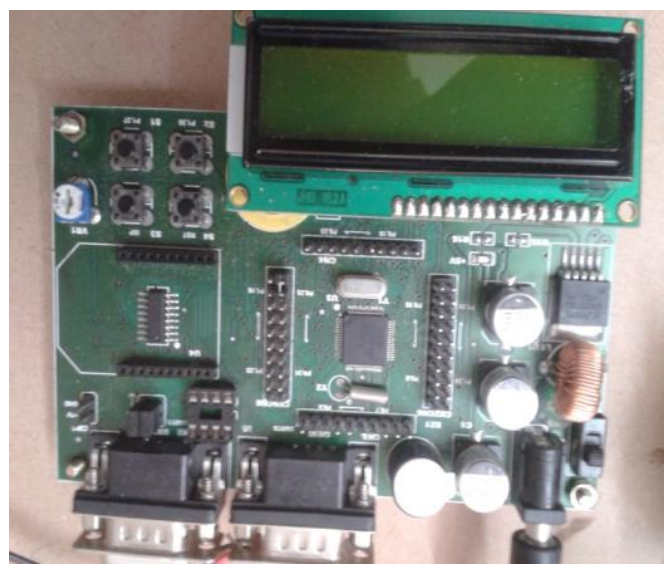

Fig 3: Sectional view of the ARM7 controller

\subsection{Buzzer}

Buzzer is a piezoelectric sound component which operates on a principle utilizing natural oscillation of piezoelectric ceramics. The sound source for piezoelectric sound component is piezoelectric diaphragm which consists of piezoelectric ceramic plate with electrodes attached on both sides. Frequency range between $2 \mathrm{khz}$ t0 $4 \mathrm{khz}$ are easily audible. So, resonating frequency of the buzzer is selected within that range itself.

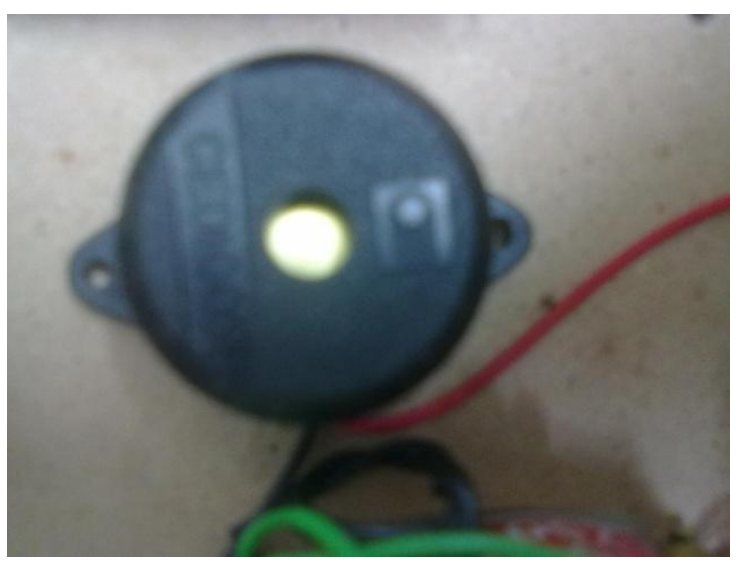

Fig 4: BUZZER to blow alarm

\subsection{Sensor}

We are using LM358 sensor for measuring the pulse rate of the person. When LED illuminate the light on the skin then there is a reflectivity caused by varying blood content of human tissues which are almost invisible are captured and pre-processed the data and forward to ARM controller

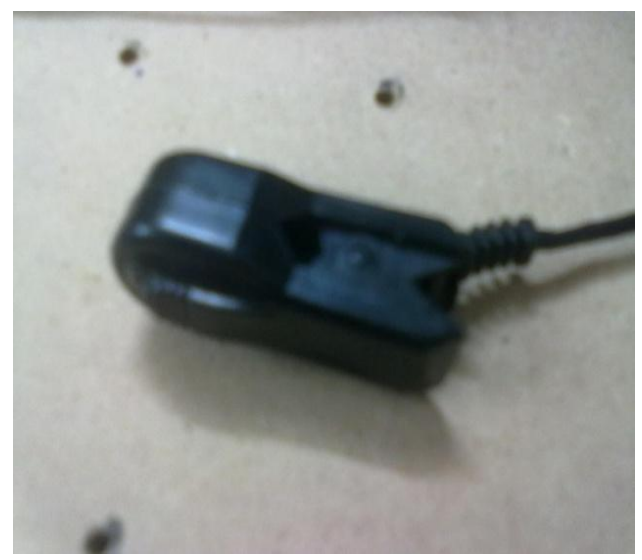

Fig 5: Pulse sensor(LM358) to sense pulse values

\subsection{GSM Modem}

Analogic's GSM Smart Modem is a multi-functional, ready to use, rugged and versatile modem that can be embedded or plugged into any application. The Smart Modem can be customized to different types of applications by using the standard AT commands. The modem is fully type-approved with all the features of Voice, Data, Fax, SMS, and Internet etc. It mainly consists of three components such as RS232, antenna, power. The frequency of antenna ranges from 900$1800 \mathrm{mhz}$. The most important component is SIM card, which has to be inserted externally. 


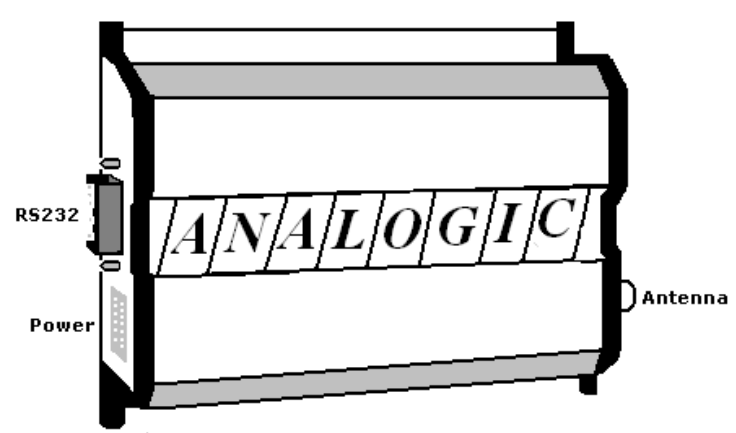

Fig 6: GSM modem(TSEL100827M1)

\subsection{Max 232}

To make communication between two electronic devices manufactured by different manufacturers Electronic Industries Association (EIA) set a slandered called Rs-232.In RS-232, a 1 is represented by -3 to $-25 \mathrm{~V}$, while a 0 bit is +3 to $+25 \mathrm{~V}$, making -3 to +3 undefined. For this reason, we use MAX232 which is a voltage converter which converts TTL level to RS-232 voltage levels and vice-versa. So here we are using this MAX-232 to have compatibility between the BUZZER and microcontroller.

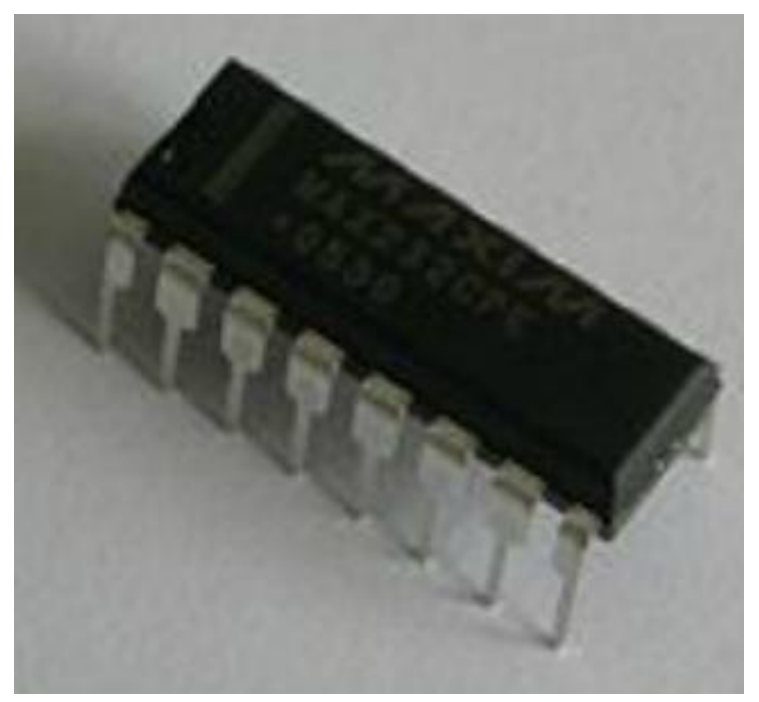

Fig 7: max-232 chip

\subsection{Zigbee}

Two electronic devices can be controlled and monitored by communicating between them. This is possible by many wireless networks among them one is zigbee. zigbee was build on IEEE 802.15.4 protocol. It defines physical layer and MAC layer above which it defines other layers also. The main advantage of zigbee when compared to other wireless network is low power consumption, low cost, high data rate. In zigbee data is transferred in the form of packets. The serial interface data rate is $1200-115200 \mathrm{bps}$, RF data rate $250,000 \mathrm{bps}$. It can handle 16 direct sequence channels and support different type of topologies like point-to-point, point-to-multipoint, peer-topeer.

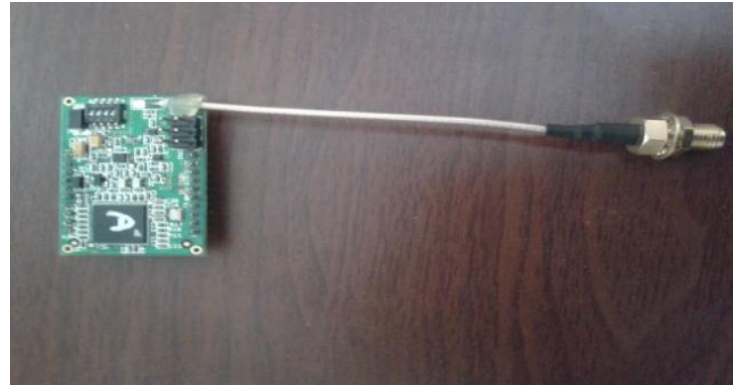

Fig 8: zigbee module

\section{DATA FLOW IN MODEL BIO- TELEMETRIC SYSTEM}

For monitoring persons vital conditions we are using different hardware elements such as ARM processor, sensor, Buzzer, GSM modem, zigbee module. Each element will operate at different voltage levels. So we use step down transformer which convert the $230 \mathrm{~V}$ to $18 \mathrm{~V}$ pulsating DC. By using bridge rectifier $1 \mathrm{~N} 4007$ diode and capacitors we convert pulsating DC into 18V DC. By rectifier 7812 we are converting the $18 \mathrm{~V}$ DC to $12 \mathrm{~V}$ DC. The filtered and regulated voltage is once again filtered for ripples using an electrolytic capacitor 100uf and then by using the variable regulator we are converting $12 \mathrm{~V} \mathrm{DC}$ to $5 \mathrm{~V}$ and $3.3 \mathrm{~V}$. All the sensors and LCD display require $3.3 \mathrm{~V}$ DC to operate. ARM controller and MEMS require $5 \mathrm{~V}$ to operate. PULSE SENSOR is connected to the port P0.7 This element will collect their respective data and forward it arm by using GPI pins. The zigbee module master is connected to UART0, GSM MODEM is connected to uart1, BUZZER is connected to the port p0.16. After preprocessing then received data is forwarded to LCD display, zigbee module. when the received value exceeds threshold value 82 , information is passed over GSM modem to the respective number. Zigbee slave which is connected at uart0 will receive the information and forward it arm controller. The information is then send to the LCD display at doctor section then the buzzer connected to port p0.16 blows an alarm.

\section{SOFTWARE USED IN THIS PROJECT}

\subsection{Keil software}

Keil software is a cross compiler. Cross compiler is nothing but working in one environment and writing code for other environment. Micro vision simulator is used because all onchip peripherals of Atmel, Philips and Samsung smart card ARM devices can be simulated using only micro vision simulator and it in cooperates project manager, editor as well as debugger in a single environment. We create source files using a text editor like notepad and each source $\mathrm{C}$ file is compiled using compiler with specific list of controls. Each assembler source file can run using specific Assemblers and we run library Manager or the Linker by specifying a special list of controls. A linker output file is converted to Intel Hex File by Running the Object HEX convertor. Thus obtained HEX file is downloaded and debugged on target hardware. KIEL can be used alternatively to create source file which automatically compiles, links and converts source file to HEX file. Finally simulates or performs debugging on the hardware with access to $\mathrm{C}$ variables and memory. In an embedded application process of creating and testing is simplified by KIEL software. 


\section{REAL TIME SNAPSHOTS}

The sensed values will be displayed on the LCD display present at the inner system. Shown in fig $9 \& 10$.

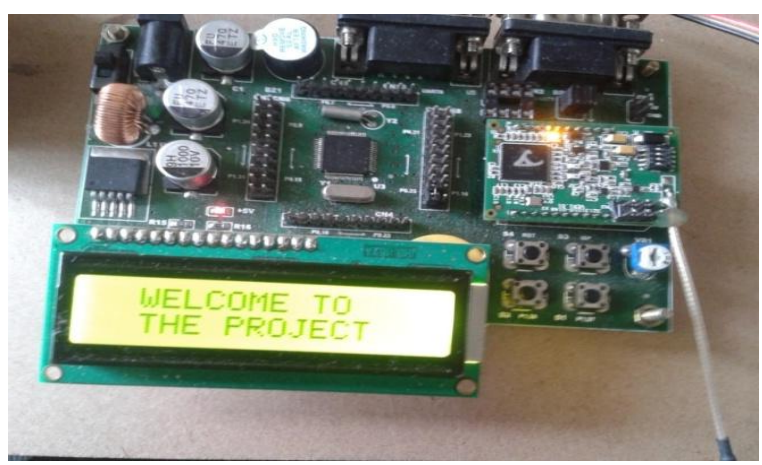

Fig 9: LCD Display

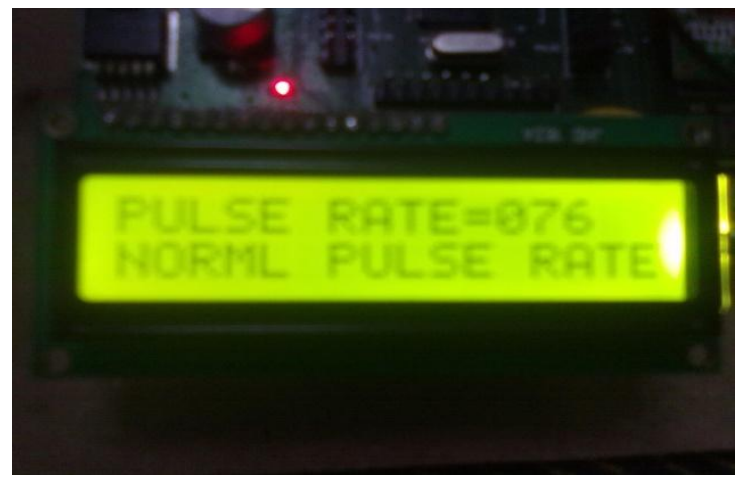

Fig 10: Display of the sensed values

Date is sent to outer section by using zigbee protocol . When the received data exceeds the threshold value (pulse $=82$ )the information is passed to receiver section as well as wireless mobile phone. The GSM will be connected shown in Figure 11.

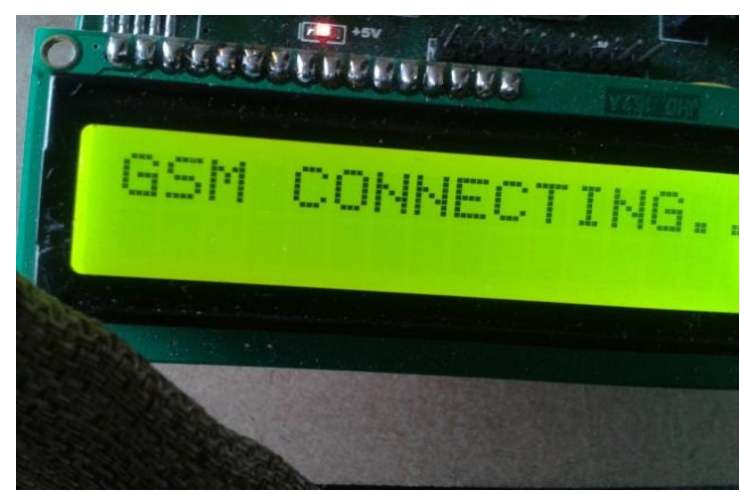

Fig 11: Represents connecting to GSM

The monitored information is transferred to the respective Mobile as shown in Figure 12.

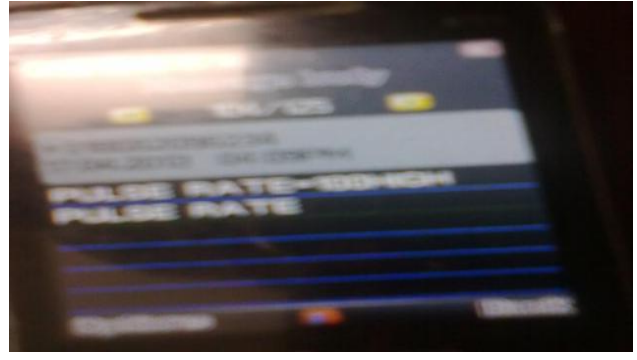

Fig 12: Display of the values on mobile phone

\section{CONCLUSION}

Bio-telemetric system is being implemented into working solutions now a days and even there is a space for improving in both concept and implementation details of this system. This system is currently designed for only indoor monitoring. With the communication implemented by $3 \mathrm{G}$ mobile technology in future this technology may include support for outdoor operation. By developing the wearable sensor we can provide service to workers who work late nights, alerts drivers and can provide emergency clinical service. Later we move further to take care of data reliability, inter relation of one problem to another with the help of expert system accepts.

\section{REFERENCES}

[1] Panfeng zhang, Kaisheng zhang "A remote sleep monitoring medical alarm system" computer communication control and automation(3CA),2010,internationalsymposium on communication networking \& broadcasting.

[2] Kang-ming, Chang,shing-hong liu "portable sleep monitoring by ECG and Accelerator and Bluetooth Transmission" $2^{\text {nd }}$ international special topic conference proceedings of the 2009 IEEE.

[3] Chang, Shih "Bluetooth based wireless sleep remote monitoring system" $6^{\text {th }}$ international special topic conference on digital object identifier in 2007.

[4] Poulito, Joshi "slep at home portable home based system for pediatric sleep apnoea diagnosis" portable information devices, IEEE conference in 2007.

[5] J.F. Pagel "obstructive sleep apnea in primary care evidence-based practice" journal of the American board of family medicine- 2007.

[6]S.J.Redmond,C.Henegha "cardiorespiratory-based sleep staging in subject with obstructive sleeo apnea" IEEE trans.biomeed.eng march-2006.

[7]Y.C.SU,Yao "signal processing inmonitoring blood oxygen concentration by LMS self-adaptive arithmetic" medical equipment journal 2006.

[8]W.Yu "Experiment study on non-invasive detection saturation saturation of blood oxygen" the military medical journal 2006.

[9] Zheng, Qiu "development of sleep monitor" foreign biomedical engineering fascicle in 2003.

[10] Wang, Chen "study on method of pulse type oxygen saturation detection" journal of medical physics in 2002.

[11]A.Q.Gu, Y.F.He "sleep analysis system" Journal of modern electrophysiology-1998.

[12] X.Z Wang, S.Q.Chen "the study of pulse type oxygen saturation detection" journal of medical physics-1995. 